\title{
n-Type Doping of Triethylenetetramine on Single-Wall Carbon Nanotubes for Transparent Conducting Cathodes
}

Rotem Dover, ${ }^{\dagger}$ Chen Klein, ${ }^{\dagger}$ Vitaly Gutkin, Johanna Zessin, Vladimir Saik, Dvir Rotem, Oded Millo, Danny Porath ${ }^{*}$, and Gabby Sarusi ${ }^{*}$

R. Dover, Dr. J. Zessin, Dr. D. Rotem, Prof. D. Porath Institute of Chemistry, and The Center for Nanoscience and Nanotechnology, The Hebrew University of Jerusalem, Jerusalem 91904, Israel

E-mail: danny.porath@mail.huji.ac.il

Dr. C. Klein, Prof. G. Sarusi

School of Electrical and Computers Engineering and Ilse Katz Institute for Nanoscale Science \& Technology, Ben-Gurion University of the Negev, Beer-Sheva, Israel

Dr. V. Gutkin

The Center for Nanoscience and Nanotechnology, The Hebrew University of Jerusalem, Jerusalem 91904, Israel

R. Dover, Prof. O. Millo

Racah Institute of Physics, and The Center for Nanoscience and Nanotechnology, The Hebrew University of Jerusalem, Jerusalem 91904, Israel

Dr. V. Saik

OCSiAl Novosibirsk. Inzhenernaya Str., 24, Novosibirsk, 630090

$\dagger$ - These authors contributed equally.

* Email: danny.porath@mail.huji.ac.il and sarusiga@bgu.ac.il 

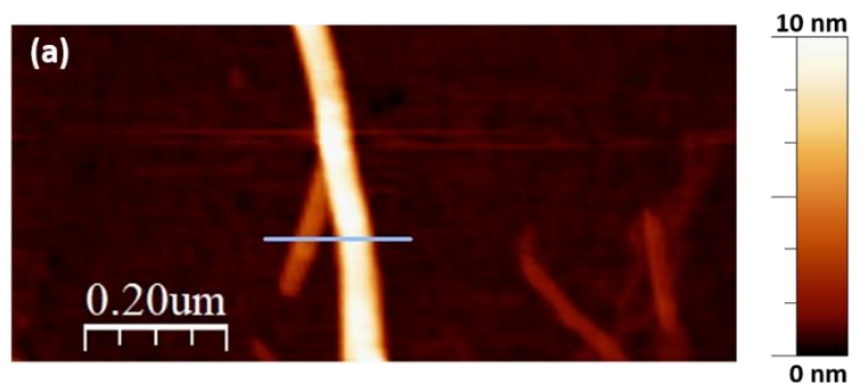

(b)

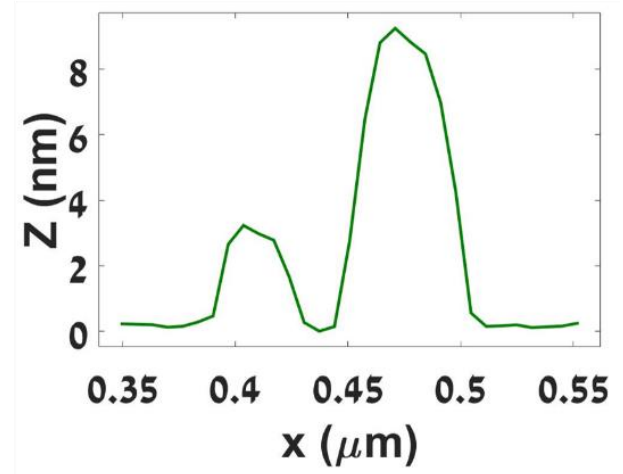

Figure S1. (a) AFM Topography scan of an exemplary junction. A cross section (grey) along the two branches forming the junction is presented in (b). Note that the thick branch is formed out of a bundle of SWCNTs and the thin is a single form of SWCNTs.
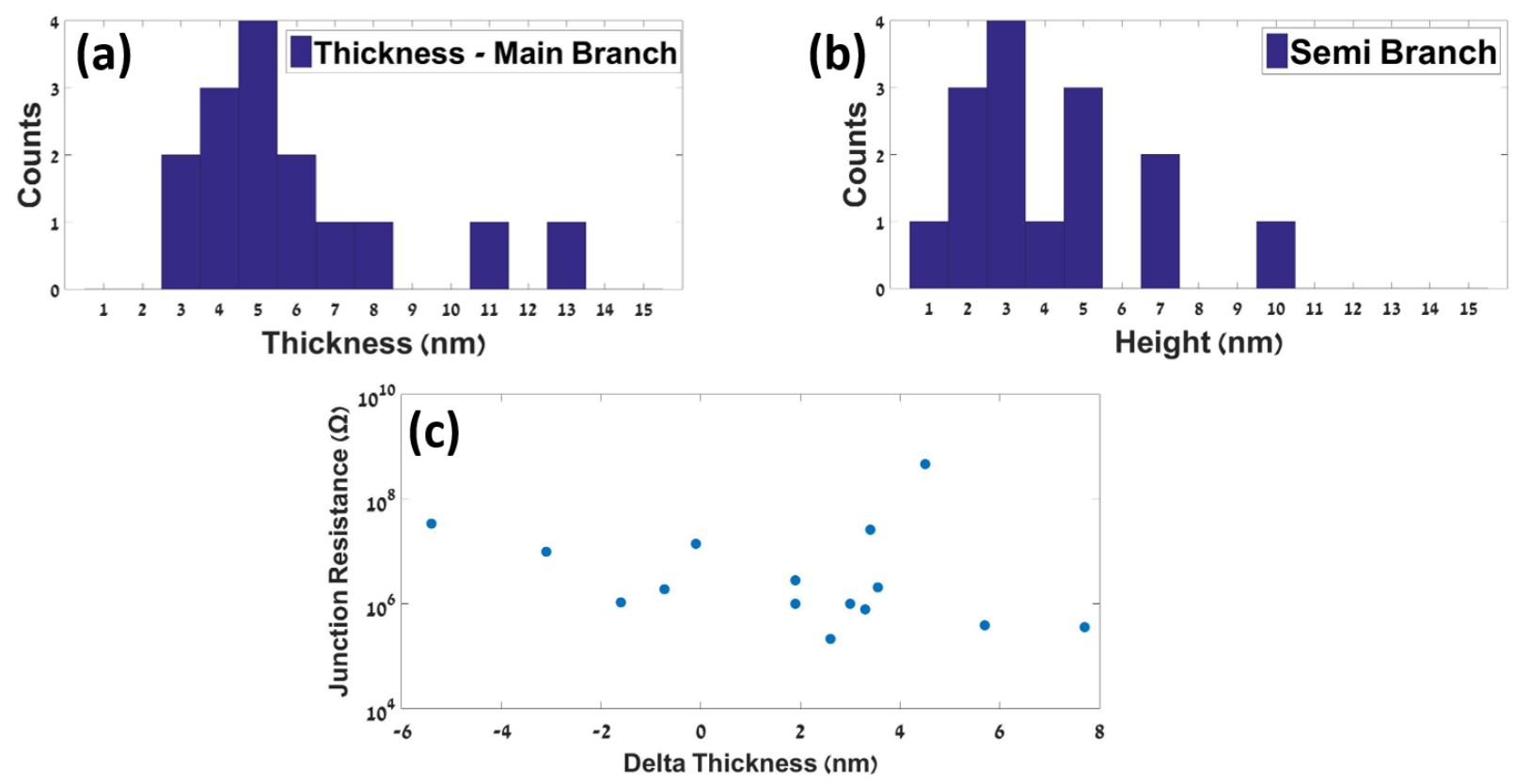

Figure S2. Thickness distribution histograms of measured SWCNT branches before (a) and after (b) an interwire for one of the samples. (C) A plot of Junction resistance as a function of the difference in thickness at each measured junction showing no dependency. 

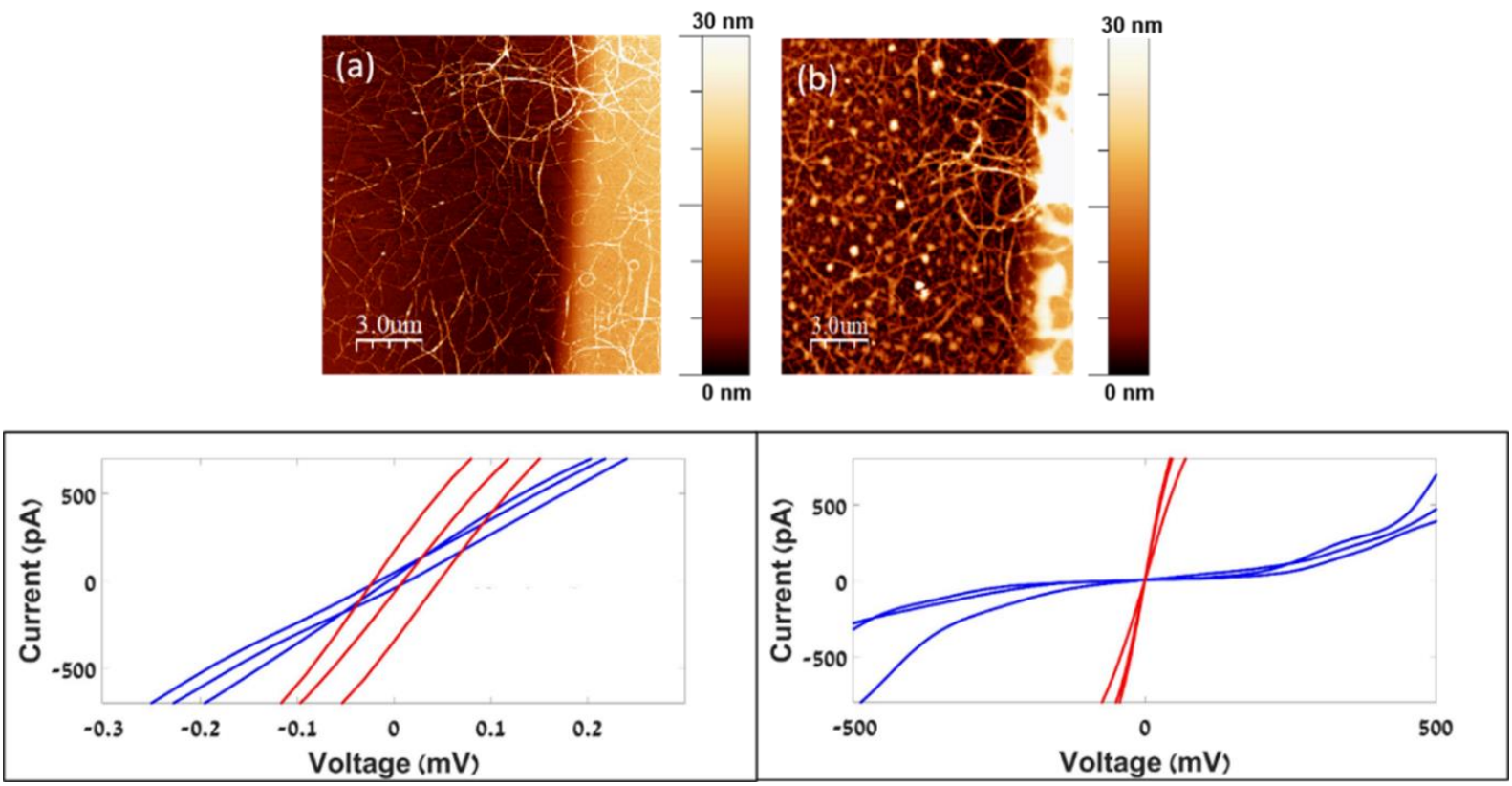

Figure S3. Excessive doping of SWCNTs by TETA. AFM topography scans of SWCNTs deposited on mica surface located at the gold border (a) before and (b) after exposure to heat and TETA fumes for 30 minutes at $120^{\circ} \mathrm{C}$ on a pre-heated plate. Conductivity measurements are exhibited below the scans respectively, showing a significant increase in contact resistance between the cAFM tip and the SWCNTs molecules and a significant increase in junction resistance following the treatment. Red (blue) curves represents resistance before (after) junction. 


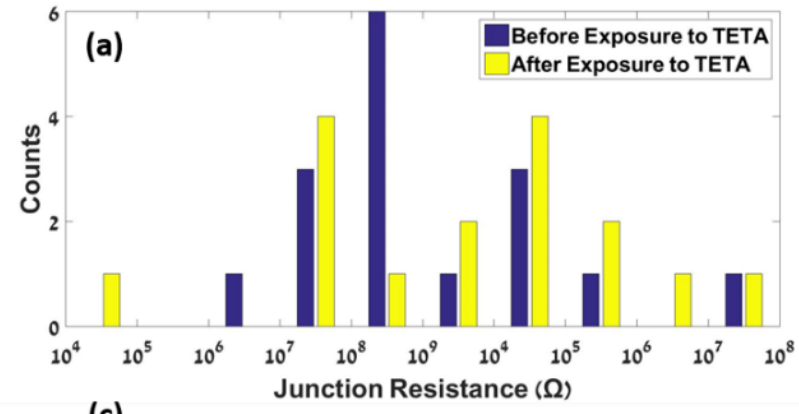

${ }_{6}$ (c)

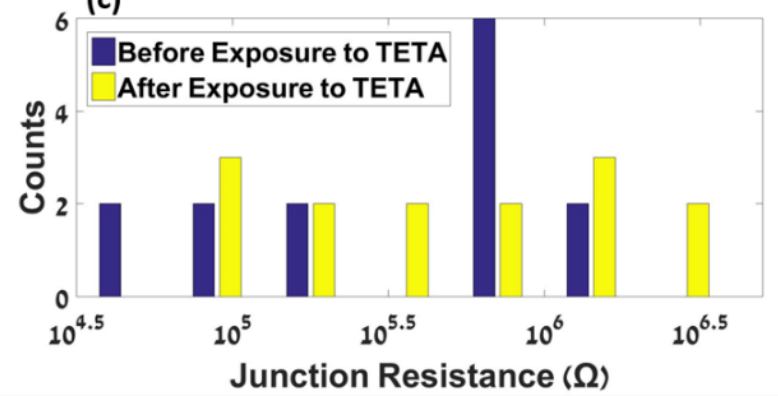

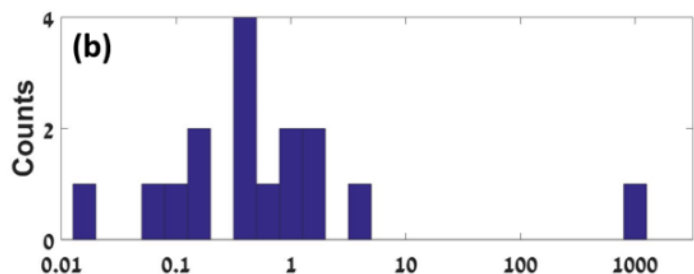

Junction resistance before any treatment $\overline{\text { Junction resistance after exposure to TETA }}$

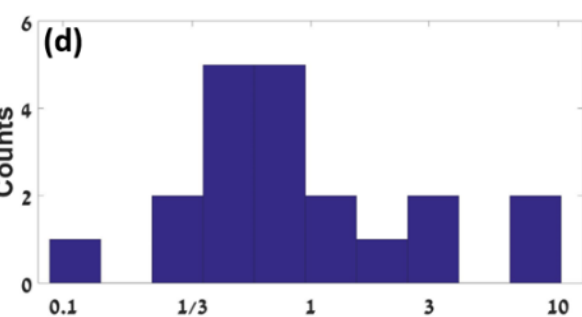

Junction resistance before any treatment Junction resistance after exposure to TETA

Figure S4. Histograms in logarithmic scales of junction resistance before any treatment (blue), after exposure to TETA fumes (yellow) of the first (a) and second (c) measured samples. (b,d repsectivley to $a, c)$ Histogram in logarithmic scale of the ratio in junction resistance for individual junctions following TETA exposure to TETA fumes. A rise in resistance is observed in most junctions following the TETA treatment. However, few junctions show an improvement.

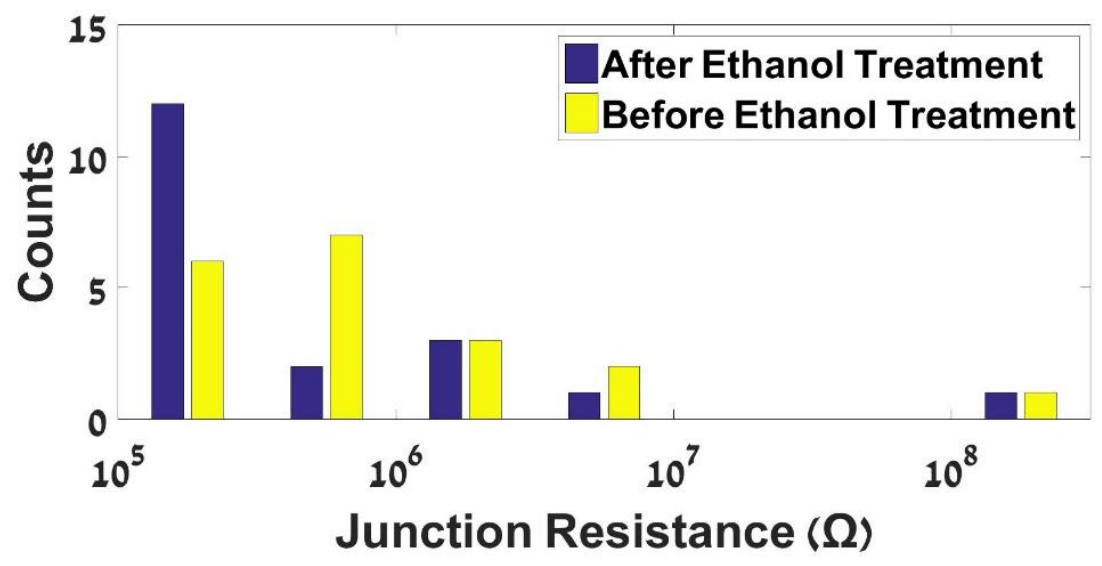

Figure S5. Histograms in logarithmic scales of junction resistance before any treatment (blue), after exposure to ethanol (yellow). 


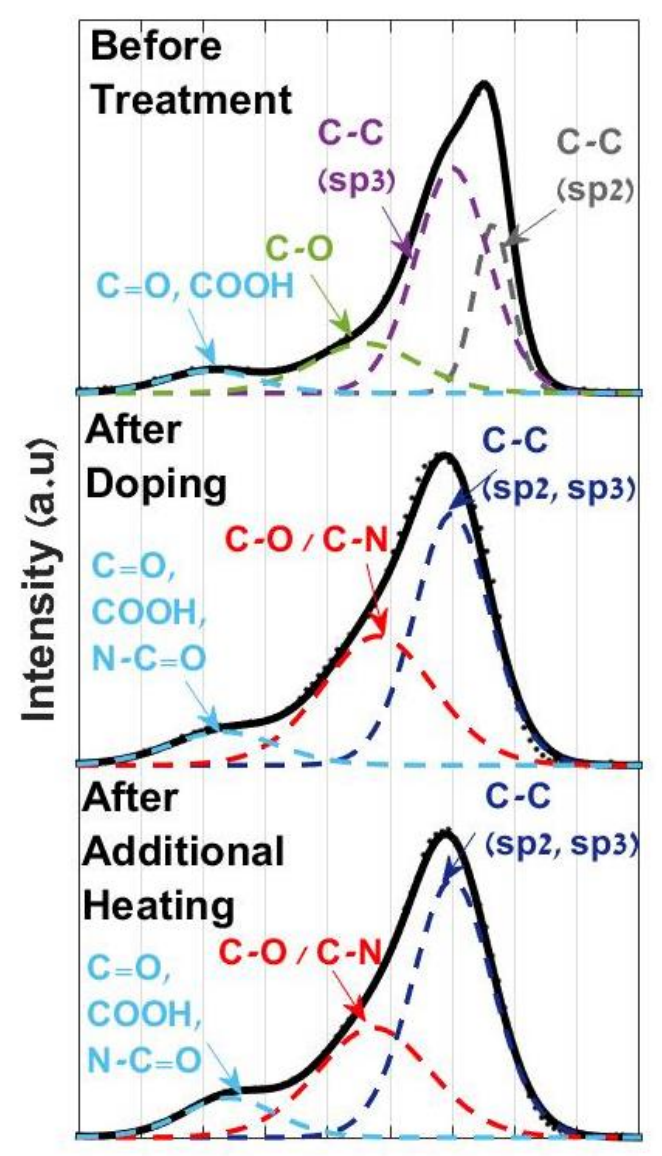

291290289288287286285284283282 Binding Energy (eV)
Figure S6 - High resolution XPS spectrums of SWCNTs before and after chemical treatments. C1s spectra of SWCNTs before any treatment, after TETA doping and after additional heating. Sum of gaussianslorentzian fitting demonstrates signals from different bonds Carbon is participated in. A low signal, attributed to sp2 C-C bonds, at $284.35 \mathrm{eV}$ (gray) is observed before treatments. After doping (also after additional heating), this signal overlaps with another signal. A signal, attributed to sp3 C-C bonds, at 285.00 $\mathrm{eV}$ (purple) is observed. This signal remains at the same position after both treatments (blue). However, it overlaps with sp2 bonds signals. Oxidized carbon of are observed at 286.41 and $288.88 \mathrm{eV}$ respectively. After TETA doping, these signals overlap with signals from $\mathrm{C}-\mathrm{N}$ and $\mathrm{N}-\mathrm{C}=\mathrm{O}$ respectively. These signals are significantly increased after TETA doping and slightly shifted to lower energies (286.20, $288.68 \mathrm{eV}$ respectively). After additional heating, the pale blue signal shifts further to lower energies (288.58 eV).

A signal from C 1s was observed for all the samples. Summation over Gaussian-Lorentzian peak fitting for $\mathrm{C}$ 1s signal was done. Gaussian-Lorentzian centers were located at the positions of known binding energies for carbon bonds. The signals at 283.99 $\pm 0.43,285.00$ (a reference value), $286.55 \pm 0.13$, and $288.81 \pm 0.12 \mathrm{eV}$ were averaged over four different measurements from undoped sample. These signals are attributed to $\mathrm{C}-\mathrm{C}$ bonds, in formation of $\mathrm{sp}^{2}$ lattice and $\mathrm{sp}^{3}$ hybridizations, and oxidized Carbon $(\mathrm{C}-\mathrm{O}$ and $\mathrm{C}=\mathrm{O} / \mathrm{COOH})$, respectively. The source of the $\mathrm{C}-\mathrm{C} \mathrm{sp}{ }^{2}$ hybridization signal is from the lattice structure of the SWCNTs. The C-C $\mathrm{sp}^{3}$ hybridization signal may originate from several sources: mainly from SDBS surfactant and contaminations by carbon adsorbed on the substrate. Defects in SWCNT's structure can also emit signals of $\mathrm{sp}^{3}$ bonds. However, the concentration of defects in SWCNT's lattices structure is relatively low. Oxidation of SWCNTs could occur during the sonication procedure, at the presence of SDBS surfactant and water. Spontaneous oxidation of any Carbon under standard conditions in ambient atmosphere can also occur. Thus, the signals from $\mathrm{C}-\mathrm{O}$ and $\mathrm{C}=\mathrm{O} / \mathrm{COOH}$ bonds can originate from various types of carbons adsorbed on the substrate. 
The $\mathrm{C}$ 1s spectra of the sample after exposure to TETA fumes shows a complete overlap between signal emitted from $\mathrm{sp}^{2}$ and $\mathrm{sp}^{3}$ hybridizations of $\mathrm{C}-\mathrm{C}$ bonds. Therefore, we cannot clearly distinguish between them by Gaussian-Lorentzian fitting. Several elements can cause this significant change. Initially, TETA molecules form $\mathrm{C}-\mathrm{C}$ bonds in $\mathrm{sp}^{3}$ hybridization, thus, increasing its signal. At the same time, the signal from $\mathrm{sp}^{2}$ hybridizations decrease because TETA molecules adsorb on the SWCNTs surface and disrupt the signal emission from them. Additionally, since nitrogen has a higher electron-negativity than carbon it will attract electrons from surrounding carbons and remaining electrons of the carbon will require higher energy to be uprooted by XPS. Thus, the signal emitted from $\mathrm{sp}^{2}$ hybridization can be shifted to higher energies and enlarge the overlap with signal from $\mathrm{sp}^{3}$ hybridization.

Following TETA doping, C 1s spectra additionally indicates changes in signals attributed to oxidized carbons. An increase in the intensity and a shift to lower binding energies was observed in both signals from oxidized carbon. The intensity increase can be mainly attributed to further carbon oxidation. The signal of $\mathrm{C}-\mathrm{N}$ bonds within TETA molecules appears at a position that overlaps with $\mathrm{C}-\mathrm{O}$ bonds. Thus, causing a slight increase in the intensity and a shift of the signal to lower binding energy (from $286.55 \pm 0.13$ to $286.20 \mathrm{eV}$ ). A small shift of the signal of $\mathrm{C}=\mathrm{O} / \mathrm{COOH}$ bonds to lower energies is observed (from $288.81 \pm 0.12$ to 288.68 $\mathrm{eV}$ ) as well. Adsorption of TETA to SWCNTs was previously suggested to take place through linkage of carboxyl groups attachment to the SWCNTs surface ${ }^{44}$. Thus, converting COOH to $\mathrm{N}-\mathrm{C}=\mathrm{O}$ by amidation is a possibility. The binding energy of $\mathrm{C} 1 \mathrm{~s}$ core shell is lower in $\mathrm{N}-\mathrm{C}=\mathrm{O}$ than $\mathrm{COOH}$. Therefore, after additional heating, no significant changes are observed (location of signals: $286.17 \pm 0.08,288.65 \pm 0.07$ averaged over three different measurements). 


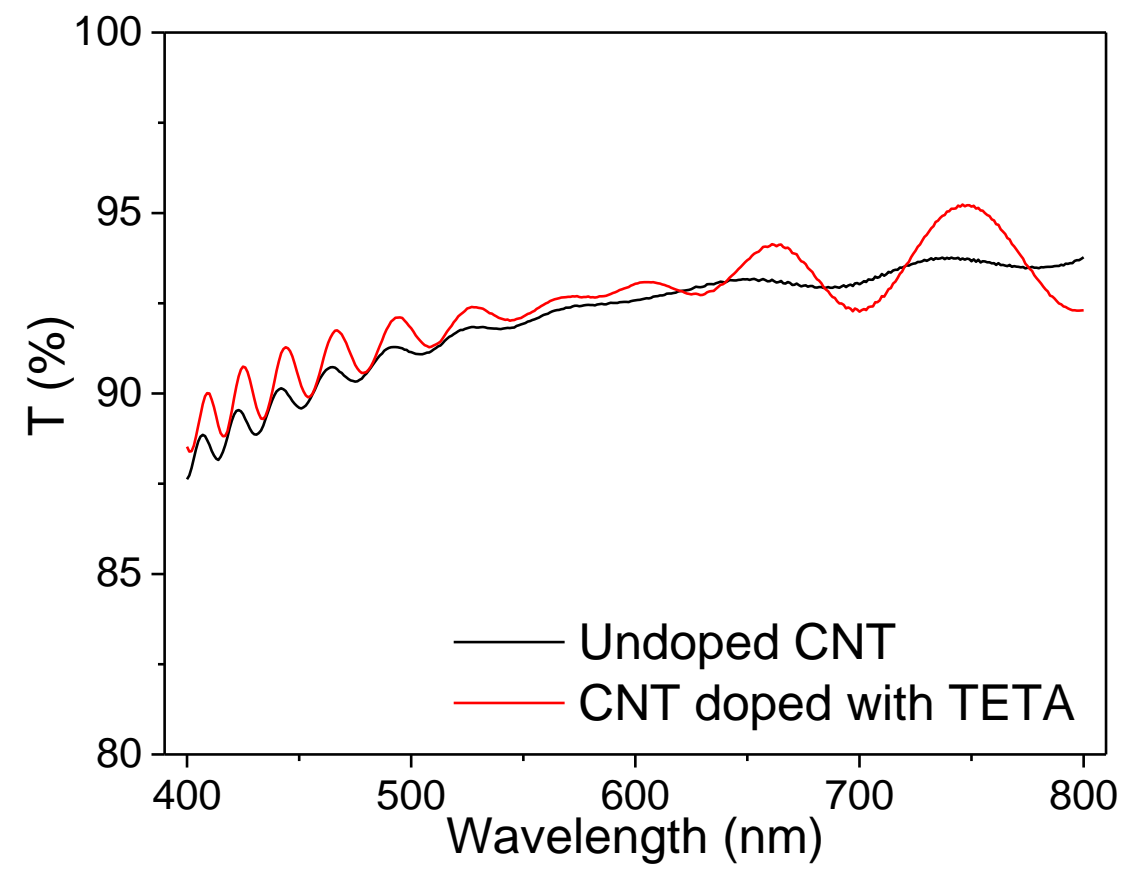

Figure S7. Optical transmission measurement by UV-Vis spectroscopy for wavelengths at the visible light spectrum for pristine SWCNTs network (black), and SWCNTs networks exposed to TETA fumes at elevated temperatures (red). 